\title{
314.
}

\section{ON THE CURVES SITUATE ON A SURFACE OF THE SECOND ORDER.}

[From the Philosophical Magazine, vol. xxII. (1861), pp. 35-38.]

A SURFACE of the second order has on it a double system of generating lines, real or imaginary; and any two generating lines of the first kind form with any two generating lines of the second kind a skew quadrangle. If the equations of the planes containing respectively the first and second, second and third, third and fourth, fourth and first sides of the quadrangle are $x=0, y=0, z=0, w=0$, and if the constant multipliers which are implicitly contained in $x, y, z, w$ respectively are suitably determined, then the equation of the surface of the second order (or say for shortness the quadric surface) is $x w-y z=0$.

Assume $\frac{y}{x}=\frac{\mu}{\lambda}, \frac{z}{x}=\frac{\nu}{\rho}$, then $\frac{\mu}{\lambda}, \frac{\nu}{\rho}$, or say $(\lambda, \mu, \nu, \rho)$, may be regarded as the coordinates of a point on the quadric surface; we in fact have $x: y: z: w=1: \frac{\mu}{\lambda}: \frac{\nu}{\rho}: \frac{\mu \nu}{\lambda \rho}$, or what is the same thing, $=\lambda \rho: \mu \rho: \nu \lambda: \mu \nu$. The four quantities $(\lambda, \mu, \nu, \rho)$ are for symmetry of notation used as coordinates; but it is to be throughout borne in mind that the absolute magnitudes of $\lambda$ and $\mu$, and of $\nu$ and $\rho$ are essentially indeterminate; it is only the ratios $\lambda: \mu$ and $\nu: \rho$ that we are concerned with.

An equation of the form

$$
(* \gamma \lambda, \mu)^{p}(\nu, \rho)^{q}=0,
$$

that is, an equation homogeneous of the degree $p$ as regards $(\lambda, \mu)$, and homogeneous of the degree $q$ as regards $(\nu, \rho)$, represents a curve on the quadric surface; and this curve is of the order $p+q$. In fact, combining with the equation of the curve the equation of an arbitrary plane

$$
A x+B y+C z+D w^{\prime}=0,
$$


this equation, expressed in terms of the coordinates $(\lambda, \mu, \nu, \rho)$, is

$$
A \lambda \rho+B \mu \rho+C \nu \lambda+D \mu \nu=0
$$

or, as it is more conveniently written,

$$
\left(\begin{array}{l}
C, D \gamma \lambda, \mu)(\nu, \rho)=0 ; \\
A, B
\end{array} \mid\right.
$$

and if from this and the equation of the curve we eliminate $\lambda: \mu$ or $\nu: \rho$, say the second of these quantities, we obtain

$$
(* \gamma \lambda, \mu)^{p}(-A \lambda-B \mu, C \lambda+D \mu)^{q}=0,
$$

which is of the order $p+q$ in $(\lambda, \mu)$; and $\lambda: \mu$ being known, $\nu: \rho$ is linearly determined. There are thus $p+q$ systems of values of the coordinates, or the plane meets the curve in $p+q$ points; that is, the curve is of the order $p+q$.

A linear equation $A \lambda+B \mu=0$ gives a generating line, say of the first kind, of the quadric surface, and a linear equation $C \nu+D \rho=0$ gives a generating line of the second kind: and by combining the one or the other of these equations with the equation of the curve, it is at once seen that the curve meets each generating line of the first kind in $q$ points, and each generating line of the second kind in $p$ points.

Consider the curves of the order $n$ : the different solutions of the equation $p+q=n$ give different species of curves. But the solution $(n, 0)$ gives only a system of $n$ generating lines of the first kind, and the solution $(0, n)$ gives only a system of generating lines of the second kind. And in general the solutions $(p, q)$ and $(q, p)$ give species of curves which are related, the one of them to the generating lines of the first and second kinds, in the same way as the other of them to the generating lines of the second and first kinds; and they may be considered as correlative members of the same species. The number of distinct species is thus $\frac{1}{2}(n-1)$ or $\frac{1}{2} n$, according as $n$ is odd or even; for $n=3$ we have the single species $(2,1)$ or $(1,2)$; for $n=4$, the two species $(1,3)$ or $(3,1)$, and $(2,2)$; for $n=5$, the two species $(4,1)$ or $(1,4)$, and $(3,2)$ or $(2,3)$; and so on. Thus for $n=3$, the species $(2,1)$ is represented by an equation of the form

$$
(a, b, c \gamma \lambda, \mu)^{2} \nu+\left(a^{\prime}, b^{\prime}, c^{\prime} \gamma \lambda, \mu\right)^{2} \rho=0,
$$

which belongs to a cubic curve in space. To show $\grave{d}$ posteriori that this is so, I observe that the equation expressed in terms of the original coordinates $(x, y, z, w)$ is

$$
\left.x(a, b, c \gamma x, y)^{2}+z\left(a^{\prime}, b^{\prime}, c^{\prime}\right\} x, y\right)^{2}=0,
$$

which by means of the equation $x w-y z=0$ of the quadric surface is reduced to

$$
\left(a, b, c \gamma(x, y)^{2}+a^{\prime} x z+2 b^{\prime} y z+c^{\prime} y w=0 ;\right.
$$

and this is the equation of a quadric surface intersecting the quadric surface $x w-y z=0$ in the line $x=0, y=0$; and therefore also intersecting it in a cubic curve. 
For $n=4$, I take first the species $(2,2)$ [the quadriquadric curve] which is represented by an equation of the form

$$
(a, b, c \gamma \lambda, \mu)^{2} \nu^{2}+2\left(a^{\prime}, b^{\prime}, c^{\prime} \gamma \lambda, \mu\right)^{2} \nu \rho+\left(a^{\prime \prime}, b^{\prime \prime}, c^{\prime \prime} \gamma \lambda, \mu\right)^{2} \rho^{2}=0,
$$

${ }_{8}{ }^{\circ}$ which in fact belongs to a quartic curve, the intersection of two quadric surfaces. For, reverting to the original coordinates, the equation becomes

$$
(a, b, c \gamma x, y)^{2} x^{2}+2\left(a^{\prime}, b^{\prime}, c^{\prime} \chi x, y\right)^{2} x z+\left(a^{\prime \prime}, b^{\prime \prime}, c^{\prime \prime} \chi x, y\right)^{2} z^{2}=0,
$$

which by means of the equation $x w--y z=0$ of the quadric surface is at once reduced to

$$
(a, b, c \gamma x, y)^{2}+2 a^{\prime} x z+4 u^{\prime} y z+2 c^{\prime} y w+a^{\prime \prime} z^{2}+2 b^{\prime \prime} z w+c^{\prime \prime} w^{2}=0,
$$

which is the equation of a quadric surface intersecting the given quadric surface $x w-y z=0$ in the curve in question.

Consider next the species $(3,1)$ [the excubo-quartic curve] represented by an equation of the form

$$
(a, b, c, d \gamma \lambda, \mu)^{3} \nu+\left(a^{\prime}, b^{\prime}, c^{\prime}, d^{\prime} \gamma \lambda, \mu\right)^{3} \rho=0,
$$

which is the other species of quartic curve situate on only a single quadric surface. Reverting to the original coordinates, the equation becomes

$$
(a, b, c, d \gamma x, y)^{3} x+\left(a^{\prime}, b^{\prime}, c^{\prime}, d^{\prime} \gamma x, y\right)^{3} z=0
$$

and by means of the equation $x w-y z=0$ of the quadric surface this is reduced to

$$
(a, b, c, d \gamma x, y)^{3}+a^{\prime} x^{2} z+3 b^{\prime} x y z+3 c^{\prime} y^{2} z+d^{\prime} y^{2} w=0,
$$

which is the equation of a. cubic surface containing the line $(x=0, y=0)$ twice, and therefore along this line touching the quadric surface $x w-y z=0$; and consequently intersecting it besides in a quartic curve. And in like manner for the curves of the fifth and higher orders which lie upon a quadric surface.

The combination of the equations

$$
\begin{aligned}
& (* \gamma \lambda, \mu)^{p}(\nu, \rho)^{q}=0, \\
& \left(*^{\prime} \gamma \lambda, \mu\right)^{p^{\prime}}(\nu, \rho)^{q^{\prime}}=0,
\end{aligned}
$$

shows at once that two curves on the same quadric surface of the species. $(p, q)$ and $\left(p^{\prime}, q^{\prime}\right)$ respectively intersect in a number $\left(p q^{\prime}+p^{\prime} q\right)$ of points. Thus if the curves are $(1,0)$ and $(1,0)$, or $(0,1)$ and $(0,1)$, i.e. generating lines of the same kind, the number of intersections is $1.0+0.1=0$; but if the curves are $(1,0)$ and $(0,1)$, i.e. generating lines of different kinds, the number of intersections is $1.1+0.0=1$.

The notion of the employment of hyperboloidal coordinates presented itself several years ago to Prof. Plïcker (see his paper "Die analytische Geometrie der Curven auf den Flächen zweiter Ordnung und Classe," Crelle, vol. xxxıv. pp. 341-359, 1847); but the systems made use of, e.g. $\xi=-\frac{d}{\mu} \frac{z}{y}, \eta=-\frac{d}{\mu} \frac{z}{x}$, with $z(z+d)+\mu x y=0$ for the equation of the surface of the second order, is less simple; and the question of the classification of the curves on the surface is not entered on.

2, Stone Buildings, W.C., May 24, 1861. 